\title{
Evaluation Model of Equipment's Operational Readiness Based on Entropy Weight Method and VIKOR Method
}

\author{
Xinpeng Wang \\ Department of Weapon Engineering, Naval University of Engineering, Wuhan 430033, China
}

1204297054@qq.com

\begin{abstract}
Keywords: Operational readiness; Entropy weight method; VIKOR method.
Abstract. To solve the problem of lacking of systematic evaluation of the equipment's operational readiness that brings much difficulty to adapt to the requirements of the current transformation and construction equipment support, an evaluation model of operational readiness integrating entropy weight method and VIKOR method are established based on the selection of the proper operational readiness index. According to the relevant information of equipment service, the model is evaluated and validated. It is proved that this method can effectively evaluate equipment's operational readiness, and the research conclusion has certain reference significance for improving equipment's operational readiness.
\end{abstract}

\section{Introduction}

Operational readiness reflects the combat effectiveness of equipment; to a certain extent determine the level of the ability of the equipment to perform tasks. Foreign countries attach great importance to operational readiness; a special operational readiness information system has been established to collect relevant information of equipment in the readiness of combat in order to provide information and evidence for the achievement of readiness objectives, the detection and improvement of operational readiness deficiencies [1].

Evaluation of equipment's operational readiness is an important part of supportability testing and evaluation, and its purpose is to measure whether the equipment meets the requirements of the operational readiness, evaluate whether the design characteristics of supportability meet the requirements stipulated in the contract, whether the planned support resources meet the specified function and performance requirements, whether the coordination between equipment support resources, whether the variety and quantity of equipment support resources meet the needs, whether the established support system meets the required support capacity; to determine the reason why the deviation from the predetermined indemnity requirement and to determine the methods of defects to correct the protection and improve the operational readiness[2].

Evaluation of equipment's operational readiness is an important and effective decision-making tool to realize the comprehensive support of equipment, which runs through the whole process of equipment development and extends to the post-deployment use stage. In this paper, according to the assessment requirements, analysis of operational readiness factors, select the relevant indicators, the entropy weight method and VIKOR method are used to evaluate the operational readiness of equipment. And then conduct validation using the relevant data service equipment validation assessment. This method is of great significance to realize the evaluation of equipment's operational readiness and optimal allocation of maintenance resources.

\section{Requirements of Equipment's Operational Readiness Evaluation}

According to the requirements of the "Comprehensive Support outline", the equipment readiness integrity assessment is divided into three stages: pre-deployment evaluation, post-deployment evaluation and follow-up evaluation.

(1) Pre-deployment evaluation. Before the deployment of the equipment, the preliminary analysis and evaluation of the system readiness and integrity of the system are carried out in the process of finalizing the design, producing the finalizing stage and the probationary period of the troops, and 
find out the problem of the operational readiness of the equipment system and take corrective measures as soon as possible.

(2) Post-deployment evaluation. Within one to two years after the deployment of the equipment, the initial assessment of the operational readiness is conducted under the conditions of the initial support capability of the troops, and problems are found and the security system of the planning is adjusted in a timely manner.

(3) Follow-up evaluation. Within three to ten years (equipment system maturity) after the deployment of the equipment, the follow-up evaluation of operational readiness is conducted. Through the assessment, further adjustment and improvement of security systems, and equipment modification and model development to provide the necessary information.

\section{The Selection Principle of Operational Readiness Index}

The principle of selection of equipment readiness indicators is the criteria that must be considered and followed in the process of determining the readiness index of weapon readiness according to the country's strategic objectives, operational requirements, technical and economic feasibility and so on. Equipment readiness indicators should follow the following principles [3]:

(1) Operational requirements traction principle: determine the appropriate indicators from the system operations and training requirements;

(2) Staged principle: revise and improve constantly at all stages;

(3) Advanced principle: meet the operational requirements though using high-tech advanced technology;

(4) Feasibility principle: taking full account of China's existing technical level and economic affordability and development cycle requirements and other objective conditions;

(5) Systematic principle: to equipment combat capability as the focus, with the principles and methods of system engineering research;

(6) Economic principle: consider the cost of each link in the whole life cycle;

(7) Verifiable principles: simultaneous verification of indicators to ensure that all indicators are verifiable.

\section{Evaluation of Operational Readiness Based on Entropy Weight and VIKOR Method}

The VIKOR approach is a multi-attribute decision-making method that can compete with each other in a compromise concept. This method is based on the ideal solution and the negative ideal solution, and the best way to compromise the alternatives by maximizing the "group benefit" and minimizing the "individual regret" with the compromise planning method as the core. It is suitable for multi-attribute evaluation of complex systems with multiple criteria and conflicting and mutually constrained [4].

There are $\mathrm{m}$ systems $\mathrm{X}_{1}, \mathrm{X}_{2}, \mathrm{~L}, \mathrm{X}_{\mathrm{m}}$ to be evaluated that can be either a number of independent weapon systems or status data of the same operational command system at different stages or periods. $\mathrm{x}_{\mathrm{ij}}(\mathrm{i}=1,2, \mathrm{~L}, \mathrm{~m} ; \mathrm{j}=1,2, \mathrm{~L}, \mathrm{n})$ is the value of the system $\mathrm{X}_{\mathrm{i}}$ to be evaluated relative to the combat readiness index $\mathrm{C}_{\mathrm{j}}$. According to the statistical results of equipment inspection records and test data, a number of factors influencing the operational readiness index are weighted, that is,

$$
\mathrm{C}_{\mathrm{j}}=\sum_{\mathrm{k}=1}^{\mathrm{K}_{\mathrm{j}}} \omega_{\mathrm{jk}} \mathrm{C}_{\mathrm{jk}}, \mathrm{j}=1,2, \mathrm{~L}, \mathrm{n} .
$$

Where $\omega_{\mathrm{jk}}$ is the weight of the influence factor of item $\mathrm{k}$ of class $\mathrm{j}$ index, to meet,

$$
0 \leq \omega_{\mathrm{jk}} \leq 1 \text { and } \sum_{\mathrm{k}=1}^{\mathrm{K}_{\mathrm{j}}} \omega_{\mathrm{jk}}=1 \text {. }
$$


In this way, the problem of warhead integrity assessment of the weapon system can be described by a multi-attribute decision model $\mathrm{D}=\left(\mathrm{x}_{\mathrm{ij}}\right)_{\mathrm{m} \times \mathrm{n}}$ :

$$
\begin{aligned}
& D=\left(\begin{array}{cccc}
x_{11} & x_{12} & L & x_{1 n} \\
x_{21} & x_{22} & L & x_{2 n} \\
M & M & M & M \\
x_{m 1} & x_{m 2} & L & x_{m n}
\end{array}\right) \\
& W=\left(\omega_{1}, \omega_{2}, L, \omega_{n}\right) .
\end{aligned}
$$

Where $\omega_{j}(j=1,2, \ldots, n)$ is the weight of operational readiness index $j$ and is satisfied $\sum_{j=1}^{n} \omega_{j}=1$.

In order to solve the multi - attribute decision - making problem, the evaluation step of weapon system readiness based on VIKOR method is summarized as follows:

Step1 : Construct the normalized decision matrix $F=\left(f_{i j}\right)_{m \times n}$, where

$$
f_{i j}=x_{i j} / \sqrt{\sum_{i=1}^{m}\left(x_{i j}\right)^{2}} \quad(i=1,2, L, m ; j=1,2, L, n) .
$$

Step2 : Determine the ideal solution $\mathrm{f}^{+}$and the negative ideal solution $\mathrm{f}^{-}$, that is ,

$$
\begin{aligned}
& \mathrm{f}^{+}=\left\{\max _{\mathrm{i}} \mathrm{f}_{\mathrm{ij}}\left|\mathrm{j} \in \alpha, \operatorname{minf}_{\mathrm{i}} \mathrm{f}_{\mathrm{ij}}\right| \mathrm{j} \in \beta\right\} \\
& \mathrm{f}^{-}=\left\{\min _{\mathrm{i}} \mathrm{f}_{\mathrm{ij}}\left|\mathrm{j} \in \alpha, \max _{\mathrm{i}} \mathrm{f}_{\mathrm{ij}}\right| \mathrm{j} \in \beta\right\}
\end{aligned}
$$

Where $\alpha$ represents the set of benefit attributes and $\beta$ represents the set of cost attributes.

Step3: Determine of the weight. The determination of the weight is directly related to the objectivity and accuracy of the evaluation results. One hand, the importance of indicators can reflect the relative importance of the objective existence of each index and attribute. The more important the attribute, the greater the weight given, and on the other hand, it can reflect the combat demand of the commander under different tasks. Therefore, this paper first determines the initial weight of each index $\lambda=\left(\lambda_{1}, \lambda_{2}, \mathrm{~L}, \lambda_{\mathrm{n}}\right)$ with the entropy weight method, and then adjusts it according to the actual situation.

$$
\mathrm{W}=\left(\omega_{\mathrm{j}}\right)_{1 \times n}=\left(\frac{\lambda_{\mathrm{j}} \rho_{\mathrm{j}}}{\sum_{\mathrm{j}=1}^{\mathrm{n}} \lambda_{\mathrm{j}} \rho_{\mathrm{j}}}\right)_{1 \times \mathrm{n}}, 1 \leq \mathrm{j} \leq \mathrm{n} .
$$

Where $\rho=\left(\rho_{1}, \rho_{2}, \mathrm{~L}, \rho_{\mathrm{n}}\right)$ represents the adjustment of the operational readiness weight according to the actual task.

Step4: According to the Lp-metric compromise plan, the system operational readiness maximization contribution index $S_{\mathrm{i}}$ and the minimization index $\mathrm{R}_{\mathrm{i}}$ are determined. 


$$
\begin{aligned}
& S_{i}=L_{1}, i=\sum_{j=1}^{n} \omega_{j}\left(f_{j}^{+}-f_{i j}\right) /\left(f_{j}^{+}-f_{j}^{-}\right) \quad i=1,2, L, m . \\
& R_{i}=L_{\infty}, i=\max _{j}\left[\omega_{j}\left(f_{j}^{+}-f_{i j}\right) /\left(f_{j}^{+}-f_{j}^{-}\right)\right] \quad i=1,2, L, m .
\end{aligned}
$$

Step5: Determine the system combat readiness value $Q_{i}$.

$$
\mathrm{Q}_{\mathrm{i}}=\frac{v\left(\mathrm{~S}_{\mathrm{i}}-\mathrm{S}^{+}\right)}{\mathrm{S}^{-}-\mathrm{S}^{+}}+\frac{(1-v)\left(\mathrm{R}_{\mathrm{i}}-\mathrm{R}^{+}\right)}{\mathrm{R}^{-}-\mathrm{R}^{+}} .
$$

Where

$$
\begin{aligned}
& \mathrm{S}^{+}=\min _{\mathrm{i}} \mathrm{S}_{\mathrm{i}}, \mathrm{S}^{-}=\max _{\mathrm{i}} \mathrm{S}_{\mathrm{i}} \quad \mathrm{i}=1,2, \mathrm{~L}, \mathrm{~m} . \\
& \mathrm{R}^{+}=\min _{\mathrm{i}} \mathrm{R}_{\mathrm{i}}, \mathrm{R}^{-}=\max _{\mathrm{i}} \mathrm{R}_{\mathrm{i}} \quad \mathrm{i}=1,2, \mathrm{~L}, \mathrm{~m} .
\end{aligned}
$$

Decision to compromise the coefficient of $v(0 \leq v \leq 1)$, take $v>0.5$ that mainly based on the system operational readiness of the contribution of the indicators to make a decision; take $v<0.5$ that the main system of operational readiness caused by the decline in the technical status indicators to make decisions; $v=0.5$ is the decision to make a compromise on both sides.

Step6: The system is sorted according to the values of $Q_{i}, S_{i}$ and $R_{i}$. First determine the three constraints:

(1) Feasible condition

$$
\mathrm{Q}\left(\mathrm{X}_{\mathrm{i}}\right) \geq \mathrm{Q}\left(\mathrm{X}_{0}\right), \mathrm{i}=1,2, \mathrm{~L}, \mathrm{~m}
$$

$\mathrm{X}_{0}$ represents the basic system which operational readiness is sufficient to meet the basic requirements of the operational mission. $X_{0}$ is usually determined based on the weapon system under the conditions of the tactical technical parameters of the lower limit and seek the views of experts or commanders. Under different task conditions, the index value covered by $\mathrm{X}_{0}$ will be some differences.

(2) Level difference condition

$$
\mathrm{Q}\left(\mathrm{a}^{\prime}\right)-\mathrm{Q}\left(\mathrm{a}^{\prime \prime}\right) \leq \frac{1}{\mathrm{~m}-1}
$$

Where a' and a" respectively represent the first and second systems to be evaluated according to the $\mathrm{Q}$ value. $\mathrm{m}$ represents quantity.

(3) Consistency condition

After sorting according to the $\mathrm{Q}$ value, the system a' also ranks the first on $\mathrm{S}$ and $\mathrm{R}$ at the same time.

Only the system that satisfies the constraint (1) has the operational readiness to perform the specified operational tasks, otherwise it needs to be further analyzed in order to implement targeted technical support. If the above three conditions are satisfied at the same time, then a' can be determined as the best operational readiness system; If both the constraint (1) and the constraint (2) are satisfied, but the constraint condition (3) is not satisfied, then a' and a" are the optimal choices; If both the constraint (1) and the constraint (3) are satisfied, but the constraint (2) is not satisfied, then $a^{\prime}, a^{\prime \prime}, L, a^{(M)}$ is the optimal choice. Where $A$ is satisfied 


$$
\mathrm{Q}\left(\mathrm{a}^{\prime}\right)-\mathrm{Q}\left(\mathrm{a}^{(\mathrm{M})}\right) \leq \frac{1}{\mathrm{~m}-1}
$$

\section{Applications}

Assuming that the baseline readiness index of a reference equipment system is 0.95 , the four systems of the equipment are evaluated by the integrated entropy method and VIKOR method. According to the influencing factors of operational readiness and the selection principle of index, four different indexes are selected: storage reliability, complexity of equipment system, reliability maintenance (RMS) improvement degree and maintenance support ability. score level of $\mathrm{x}_{\mathrm{ij}}$ is divided into 4, respectively, corresponding to $(4,3,2,1)$.The higher the score, the greater the degree of impact, then the system $X_{i}$ relative to the operational readiness index $C_{j}$ as shown in Table 1 below.

Table 1 System $\mathrm{X}_{\mathrm{i}}$ relative to the operational readiness index value $\mathrm{C}_{\mathrm{j}}$

\begin{tabular}{cccccc}
\hline & \multicolumn{4}{c}{ Operational Readiness Index } & \\
\cline { 2 - 5 } System & $\begin{array}{c}\text { storage } \\
\text { reliability }\end{array}$ & $\begin{array}{c}\text { complexity } \\
\text { of system }\end{array}$ & $\begin{array}{c}\text { RMS } \\
\text { improvement } \\
\text { degree }\end{array}$ & $\begin{array}{c}\text { maintenance } \\
\text { support } \\
\text { ability }\end{array}$ & Comment \\
\hline X1 & 3 & 2 & 1 & 4 & \\
X2 & 4 & 3 & 1 & 2 & $\mathrm{x}_{\mathrm{ij}}=[4,3,2,1]$ \\
X3 & 4 & 1 & 2 & 3 & \\
X4 & 4 & 2 & 1 & 3 & \\
\hline
\end{tabular}

Calculate the weight of each index according to formula (6):

$\omega_{1}=0.375, \quad \omega_{2}=0.2$

$\omega_{3}=0.125, \quad \omega_{4}=0.3$

Take $v=0.5$ to calculate the operational readiness value of four systems:

$Q_{1}=0.989 \quad Q_{2}=0.954$

$Q_{3}=0.951 \quad Q_{4}=0.978$

It can be seen from the calculation results that the four systems to be evaluated meet the conditions (1) and (2), but not the condition (3), then the system 1 and the system 4 are the optimal choice.

\section{Conclusions}

The entropy weight evaluation method determines the weight coefficient of the index according to the degree of the difference of the index value, and has certain objectivity, but ignores the subjective factors. The evaluation result is often one-sided. Therefore, the entropy weight assessment method is usually combined with other subjective evaluation methods. The VIKOR approach is a multi-attribute decision making method that can compete with each other in a concept of compromise. Although the combination of the two methods to a large extent to solve the problem of equipment's operational readiness assessment, but not a good reflection of the comprehensive protection of weapons systems and task profiles of various activities.

\section{References}

[1] Y.S. Li, H.P. Shi: submitted to Electronic Product Reliability and Environmental Testing (2007)

[2] T.X. Xu, J.T. Zhao: submitted to Aerodynamic Missile Journal(2001) 
[3] C. Dong: submitted to Ship Electronic Engineering(2015)

[4] M. Gul, E. Celik, N. Aydin and A.T. Gumus:submitted to Applied Soft Computing(2016) 